\title{
Skillful Coaching: New Directions In Teaching Health Assessment
}

\author{
Joyce Zurmehly (E-mail: zurmehly@ohiou.edu.), Ohio University \\ Mary Lynd (E-mail: mary.lynd@wright.edu.), Wright State University \\ Camille Leadingham (E-mail: leadingham@ohiou.edu.), Ohio University
}

\begin{abstract}
The purpose of this study was to examine differences in nursing student's assessment skills before and after the implementation of a cognitive apprenticeship didactic approach including thinkaloud and critical dialogue. In the nursing care of clients, a through and accurate holistic health assessment is essential. The content of the assessment task is taught in all nursing programs. Students must be competent not only in performing the examination but also in evaluating the information obtained and integrating it with the health history and lab findings. This extensive criteria makes assessment a challenging skill to master. Problems arise when students in nursing attempt to apply the theoretical assessment approaches to the clinical setting with real clients. Consequently, student's performance outcomes are often unsatisfactory, as the teaching format usually focuses on the students' acquisition of domain knowledge and psychomotor skills rather than on shaping the thinking processes involved. Students' common problems with physical assessment shows that they struggle to manipulate the abstract positions involved.
\end{abstract}

\section{Introduction}

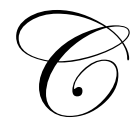

linical assessment is an important aspect of professional nursing and guides the nurse in assimilating, retrieving, and/or discarding components of information. With clinical assessment the nurse analyzes information and ultimately reaches decisions regarding client care. Unfortunately, as a result of multiple changes in health care and the current nursing shortage, nurses have increased responsibility and accountability. As a result, nurses endure not only time constraints when utilizing assessment strategies but have fewer resources available for this process. Nurses have expanded roles into advanced practice that have increased the critical decisions that must be made in caring for the client, the family, and/or the community. High client acuity and complex technology once distinguished the roles and responsibilities of the acute care nurses from that of the community care nurses. However, this distinction is now blurred as high-acuity clients have moved out of the acute settings into the community setting. Consequently, more and more nurses, both acute care nurses and communitybased nurses, are required to make complex decisions under conditions of uncertainty and risk (Cleveland \& Gilbert, 2001). Graduate nurses and nurses orientating to new positions have limited time to learn institutional policies, procedures, and standards before assuming the full responsibility for total client care. Furthermore, there are fewer experienced nurses available to act as mentors for new graduate nurses.

Excellent clinical assessment skills are essential to strengthen nursing practice as a means of increasing the accuracy of decisions and, thereby, assuring positive client outcomes. The findings from clinical assessment research can also be incorporated into nursing education to better prepare novices to manage client information and make better-informed decisions.

\section{Research Problem}

In nursing a through and accurate physical assessment is a fundamental, foundational skill in the care of clients. The content of the assessment task is taught in all nursing programs. However, student performance outcomes are often negated or unsatisfactory, as many teaching formats usually focuses on students' acquisition of 
domain knowledge and psychomotor skills rather than on shaping the process of critically thinking about the assessment data. In this research study the utilization of a cognitive apprenticeship approach coupled with a teaching methodology, which is described as think-aloud, is presented. This methodology may be useful in facilitating students in developing accurate and advanced level physical assessment skills.

\section{Cognitive Apprenticeship Theory}

The nursing literature does not appear to critically delineate the best methodology for teaching nursing assessment. Tradition conveys the theoretical and heuristic skills of physical assessment, mainly focusing on the data to be obtained. A different approach becomes evident when utilizing the theory of cognitive apprenticeship. According to this conceptual framework, just as motor skills can be taught by example, critical thinking skills can also be taught by making them accessible to the novice (Rogoff, 1990). Cognitive apprenticeship theory has been termed social constructivist because it postulates that individuals actively construct knowledge and negotiate meaning as a result of social interaction (Phillips, 1995).

Lev Semyonovich Vygotsky (1896-1934) was an important Russian psychologist who had a deep appreciation for both developmental and environmental forces that contribute to cognitive development (Berk \& Winsler, 1995). Vygotsky had a great desire to find a better way to understand the difficulties of the current social and educational problems. Vygotsky distinguished between higher and lower mental functions (Nicholl,1998). Lower mental functions are elementary and are those that are genetically inherited such as our natural mental abilities. Vygotsky was influenced by the writings of Karl Marx and theorized that higher mental functions are developed through cultural and social interactions.

Vygotsky is best known for his work on social cognitive learning model. In the social learning model Vygotsky believed that culture is the prime determinate of individual development and that humans are the only species that have a developed culture (Berk \& Winsler, 1995). Therefore, the learning development of a child is largely affected by the culture. It is through culture that a child develops their thinking and knowledge. The culture that surrounds a child provides a means for their thinking. Voygotsky believed that a culture teaches a child what to think and how to think. He used the term signs for the various tools, that cultures produce, and people use to aid their thinking and behavior (Vygotsky, 1934/1986).

Vygotsky suggested that the earliest psychological tools were memory aids and one of the single most important memory aids is speech (Vygotsky, 1934/1986). According to Vygotsky, language is the center of development and made possible because of our culture. The ability to learn language is brought about by social processes and is the organization of thinking (Voygotsky, 1934/1986). The first stage of speech is social speech or inner speech (Vygotsky, 1934/1986). Speech is used to help control behaviors of others and help overcoming difficulties. Contrary to Piaget's beliefs, Vygotsky theorized that the concepts of speech develop from external or social influences rather than biological tendencies. In this stage a child uses speech to control the behavior of others such as a statement like "I want to eat." (Vygotsky, 1934/1986). This stage is highly public or external.

The second stage is egocentric speech; this stage transfers speech from a social activity to an individualized level. It is connected to a child's thinking because it helps them overcome difficulties. During this stage, a child will talk out loud while performing certain tasks such as staking blocks. They reason and must speak it as they are doing it. This makes them reason that language must be spoken if it is to direct their behavior.

The final stage is inner speech. This is a soundless speech and is used by older children and adults. Vygotsky theorized that egocentric speech has a genetic connection with inner speech. He believed that inner speech is for oneself and social speech is for others. Vygotsky disagreed with Piaget about egocentric speech. Piaget believed that as a child overcomes egocentric speech it simply dies out and is useless, whereas Vygotsky argued that it does not just fade away. Egocentric speech simply turns into inner speech a silent dialog that we often have with ourselves when we try to problem solve (Vygotsky, 1934/1986). Once this stage is obtained one will begin to engage in all forms of higher mental functions such as counting in ones head or using logical memory. 
Vygotsky placed emphasis on the importance of a child's development and school instruction. He believed that cognitive development results from a process whereby a child learns through problem-solving experiences that are shared with someone else, this is usually a parent, teacher, sibling, peer, or mentor. Language is the primary source of interaction as the adult or mentor transmits knowledge that exists in culture to a child. As this teaching/learning process progresses the child's own language becomes his/her primary tool of adaptation. This results in the child using internal language to begin and direct behavior. Vygotsky believed a difference existed between what a child could do on their own and what a child could do with help. He called this difference the zone of proximal development. This is a distance between the actual development level at present and the potential as determined by problem solving under the guidance of an adult or mentor (Vygotsky, 1934/1986).

Vygotsky thought spontaneous development was important. He argued that if a child were a product of their own discoveries they would not advance very far. Children benefit from the knowledge and concept tools handed down by their cultures and this usually occurs in schools (Bae, 1999). Instruction therefore propels the mind forward enabling the learner to open new paths. Vygotsky both started with the same basic theory on the child as a biological organism. Vygotsky devised theories with natural and social concepts regarded neither as sufficient by itself to explain development and stressed the social side. Each is important and cognitive change cannot be understood without both. Also, Vygotsky lived in an era of state controlled Communist government; ideas upon intellectual thought were controlled, while Gilman's work reflects the intellectual environment of a free society.

Therefore since children learn through interaction, a school curriculum should be designed to contain interaction between learners and learning tasks. Most schools have an evaluation system that consists of standardized achievement tests. This method of evaluation only measures the present state of a child's development not the potential to which they can develop. Vygotsky believed that before a child could perform a task alone they need to perform this task while receiving guidance or support in order to advance (Vygotsky, 1934/1986). Assessment tools need to take into account the zone of proximal development. What a child can do on their own compared to what children can do with help. Whereas two children may have the same level of actual development but when given adult help one might solve many more problems than the other.

Using Vygotsky's theory educators need to gear lessons that stimulate, challenge, and engage a learner and then allow the learner to actively solve these challenges on their own. Although Vygotsky's theory focused on children, the concepts proposed are applicable to the learning of assessment skills by student nurses who are actually at the 'child's or beginner's level of development' in the nursing profession.

\section{Think-Aloud In Cognitive Apprenticeship}

In a cognitive apprenticeship approach, the expert's thinking aloud provides the beginner with access to both overt aspects of the skill and the more hidden inner processes of thought (Rogoff, 1991). Think aloud is a qualitative method of collecting verbal data about cognitive processes during a problem task (Erickson \& Simon, 1993). It is based on the following assumptions: 1) human cognition is information processing, 2) cognitive processes can be verbalized, 3) thinking aloud does not alter the sequence of thought processes, and 4) verbalization reflect information that is being attended to or concentrated on (Tilley, 2001). Verbal reports may reflect retrospective or concurrent thinking. Retrospective verbalization provides an oral recollection of thinking that occurred in the past. The data rely on memory and thus the longer the time period from thinking to the verbal report the greater the probability the memory will be incomplete, alter by time, or difficulty to retrieve. Concurrent verbalization is an oral report of thinking as it occurs, and is considered to produce more accurate, complete, and consistent data about a subject's reasoning (Hockly, 2000). The validity of think aloud data is also enhanced by limiting directions and verbal prompts to subjects.

\section{Physical Assessment}

Client physical assessment is a cognitive task for measuring of nursing clinical reasoning skills. In this investigation, assessment indicated the initial encounter the nursing student had with the client. Client assessment has been described as the first of many steps of the nursing process that requires reasoning skills. Assessment 
includes gathering and organization of client data, cues, or information into categories for further assimilation (Solomon, 1990). Physical assessment is the detailed examination of a client for the purpose of further analysis. The nursing function included four techniques: inspection, palpating, and auscultation. Together with the health history and laboratory measurements, the physical assessment provides information, which forms the foundation for all decisions regarding the choice and implementation of nursing interventions (Wales \& Skillen, 1997). It is this first step in the nursing process that provides the pivotal framework for all subsequent phases such as planning, intervention, and evaluation. The quality of the assessment data is imperative for effective evidence-based practice and positive health outcomes.

The assessment task consists of two distinct components--the collection of the data and the subsequent analysis of the data. The nurse must be able to accurately interpret and verify the data that is acquired during the physical examination and integrated with the health history and lab components. This integration makes assessment a challenging task for students in nursing to learn. Students must observe and possess a strong knowledge base that will enable them to distinguish between pertinent and irrelevant information. Students must be able to validate normal values and analyze the data in a manner that will culminate in a relevant plan of care for the client. In addition to cognitive and psychomotor skills, a physical assessment requires the development of affective skills for the development of appropriate and empathetic behaviors toward the client being examined. According to Milner and Collins (1992), competency in client assessment is becoming increasing important. Decisions are often critical in terms of client outcomes. Nursing educators have the responsibility of preparing student who are to perform the physical assessment within a diverse array of culturally diverse clients and health care settings and enable the student to perform the assessment skills that are deemed necessary to practice as a competent professional.

\section{Teaching Physical Assessment}

Physical assessment is taught in all nursing education programs and is included in standard nursing curriculum. Frequently used standardized assessment guides such as Functional Health Patterns by Gordon (1999) provide outlines of client functions such as perception, elimination, or coping with stress (Solomon, 1990). Other approaches follow an anatomical head-to-toe patterns or body systems patterns. The overall commonality is providing students with the information leading to obtaining the correct data in a systematic, orderly manner. Many current textbooks provide basic assessment information and many are solely dedicated exclusively to the subject. However, problems arise when students apply the theoretical assessment approaches in the clinical setting with real clients. Nursing students tend to make common errors such as missing data cues, misinterpreting data collection, prioritizing incorrectly, failing to base nursing responses on the assessment data, and neglecting the psychosocial component of client assessment (McHolm \& Geib, 1998). Consequently, these types of errors in judgment can have dangerous outcomes in today complex health care environments.

\section{Review Of The Literature}

There is limited research suggesting methodologies in teaching nursing health assessment. An inadequate number of research suggests teaching health assessment utilizing the medical model and proposing the utilization of live simulation by healthy persons who are trained to act in specified roles of assigned sick patterns. Even though mandatory physical assessment courses are evident in many schools of nursing. limited research has been performed detailing the best method to utilize. A survey performed by James and Reaby (1998) concluded that the teaching of physical assessment is in the process of evolution and continued improvement in a clear assessment pedagogy is needed. There is a clear lack of current projects and little evidence to overall success in teaching physical assessment effectively.

\section{Physical Assessment And Thinking Skills}

It is apparent that expertise in assessment skills include more than just the physical assessment evaluation criteria. The health status of patients often changes rapidly and the nursing assessment has to be an iterative ongoing process throughout the nurse-client interaction. Students in nursing struggle to manipulate the abstract propositions involved. When they enter a nursing course they have had some experience in collecting data, but as 
they acquire increasing domain knowledge they are expected to choose pertinent data and prioritize it. However. identifying critical elements and relations is not explicitly taught in many nursing courses. Thus, an important learning element is to determine connections between data.

Nursing educators have become aware of the need to address students' thinking skills, and numerous modifications to traditional teaching emphases are currently underway. Some nursing texts focus on problem solving whereas others center around critical thinking. Watson and Glaser (1964) described critical thinking as an attitude of inquiry, supported by a knowledge base and enhanced by skill in application. Improved thinking skills are expected to translate into better planning of action and improved practice. However, the format for physical assessment remains an exhaustive list of abnormalities the nursing student look for using classic examination techniques. The usual instructional approach is to point out what information is to be obtained and then to evaluate the student's written assessment report for thoroughness in presenting the data.

\section{Research Design}

The purpose of this study was to examine the differences in a Maternity nursing students' assessment skills before and after the implementation of a cognitive apprenticeship didactic approach including think-aloud and critical dialogue were implemented. A quasi-experimental design with a pre-test and post-test method was used to examine the difference in assessment skills. The research subjects were second-year nursing students enrolled in a Maternity-Child nursing course at a university college branch campus. The group consisted of 31 associate degreenursing students enrolled in the fall quarter. All participation was voluntary, and the students were assured of anonymity, safety, and their academic standing would not be affected by their participation in or withdrawal from this project. This assessment was initiated week 10 of the quarter. The student receive didactic instruction between theory and clinical. The teacher-researchers used think-aloud in teaching these students the physical assessment of the expected mother. This took place in sequential steps. A prerequisite for the students was the tasks of reading assigned chapter throughout the quarter in their obstetric textbooks. In the nursing laboratory at the college, students worked in groups of three. The student performed an assessment utilizing the head-to-toe assessment tool. The students in the lab setting performed the initial assessment. Following the first assessment the teacher demonstrated physical assessment procedures with detailed explanations of the content to be observed. The assessment was than modeled by the student with concurrent verbalization of the thinking processes involved as data was gathered and decisions for action were given. Then the students and the teacher jointly worked through other client assessment processes using consistent verbal articulation with peers observing and participating in the verbalization interpretation and prioritizing of data.

The students were encouraged to verbalize everything they noted about the client and all thoughts they had regarding what the data may indicate. In addition, they were given a problem-focused assessment to perform on a client. They were asked to say out loud any thought regarding possible nursing actions related to the data and to note any data that would still need to be obtained at another time or from another source. The teachers coaching and guidance supplemented these comments. As the students' competence increased during the assessment, the teacher withdrew the coaching support. Finally, the students independently performed another assessment using continuous dialogue. This post-test followed the same guideline as the pre-test. The teacher observed and listened to their assessment, noting any difficulties, the accuracy of the results, and the students' report.

\section{Results}

Thirty-one students completed the assessment instruments. All student participated in the think-aloud assessment intervention. Post-test scores of each assessment and for total scores were significantly higher ( $\mathrm{p}=.000)$ than pre-test scores. Total scores on the pre-test was $m=84.82$ where as on the post-test they were $m=90.46$. Overall scores were lowest on the antepartum pre-test $(m=80.1)$, while the scores for the antepartum and interpartum were similar. A similar pattern occurred in the post-test scores (Table1). 
Table 1: Pre-test And Post-test Scores (n=31)

\begin{tabular}{|l|r|r|r|c|c|}
\hline \multirow{2}{*}{ Area } & \multicolumn{2}{|c|}{ Mean score } & \multicolumn{2}{|c|}{ SD } & Significance \\
\cline { 2 - 6 } & Pre-test & Post-test & Pre-test & Post-test & \\
\hline Antepartum & 80.11 & 88.45 & 8.73 & 7.12 & .000 \\
Intrapartum & 86.77 & 91.04 & 6.44 & 5.68 & .000 \\
Postpartum & 87.59 & 91.89 & 6.59 & 4.40 & .000 \\
Total & 254.47 & 271.38 & 21.76 & 17.2 & .000 \\
\hline
\end{tabular}

In order to emphasize thinking not only about the data but also about the thinking processes themselves, the teacher and students engaged in joint and individual reflection on actions taken. The student's impressions of the various physical assessment procedures were compared and discussed. The students were asked to report how they felt about the assessment, whether they understood the significance or specific data noted, what nursing actions they would undertake and what questions they still may have. This encouraged students to identify their own gaps in domain knowledge and discuss resources that may help supplement information.

\section{Conclusions}

Analysis of the data revealed that the use of think-aloud and critical dialogue created a positive learning environment, which embodied the main characteristics of cognitive apprenticeship. Students reported increased self-confidence and identified the crucial role of peer participation. Their assessment skills improved and included the transfer of think-aloud critical dialogues to silent assessment. The significance of the results of the think-aloud process supported the theoretical framework proposed for the study emphasizing the use of this theory for promoting critical thinking in all populations.

\section{References}

1. Berk, L.E., \& Winsler, A. (1995). Scaffolding children's learning. Washington, DC: National Association Press.

2. Cleveland-Grance, B., \& Gilbert, L.H. (2001). "Connecting classroom clinic, and community: Pediatric nurse practitioner student and faculty assessment", Journal of Nursing Education, 40(4), 187-190.

3. Hockly, N. (2000). Modeling and “'Cognitive Apprenticeship' in teaching education”, ELT Journal, 54 (2), p. 118-25.

4. Nicholl, T. (1998). Vygotsky. [On-Line]. Available: http://www.massey.ac.nz./Lock/virtual/trishvyg.htm.

5. Phillips, D.C. (1995). "The good, the bad, and the ugly: The many faces of constructivism". Educational Researcher, 24 (7), 5-12.

6. Rogoff, B. (1991). "Social interaction as apprenticeship in thinking: Guidance and participation in spatial planning", in L. Resnick, J. Levine, \& S. Teasley (Eds.), Perspectives on socially shared cognition (pp. 349-364). Washington, DC: American Psychological Association.

7. Solomon, J. (1990). "Physical assessment skills in undergraduate curricula", Nursing Outlook, 38 (4), 194195

8. $\quad$ Tilley, C.L. (2001). “Cognitive Apprenticeship”, School Library Media Activities Monthly, 18 (3), 37-39.

9. Wales, M.A., \& Skillen, D.L. (1997). "Using scenarios as a teaching method in teaching health assessment”, Journal of Nursing Education, 36 (6), 256-262.

10. Vygotsky, L.S. (1986). Thought and language (A. Kozulin, trans). Cambridge, MA: MIT Press. (Original work published 1934). 